\title{
THE EFFICIENCY OF VEHICLE MONITORING LOCATIONS FOR A VOLUNTARY TRAVEL DEMAND MANAGEMENT PROGRAM
}

\author{
Joonho Ko ${ }^{1}$, Daejin $\mathrm{Kim}^{2}$, Heung Gweon $\mathrm{Sin}^{3}$, Seungjae Lee ${ }^{4}$ \\ ${ }^{1,2}$ The Seoul Institute, South Korea \\ 3,4 University of Seoul, South Korea
}

Submitted 30 December 2013; accepted 9 July 2014

\begin{abstract}
As many people are concerned about sustainable urban transportation systems, Travel Demand Management (TDM) is getting more attention as a viable option to reduce automobile dependency on an efficient way. Especially, voluntary participation-based TDM by offering incentives has been applied in many cities in recent years. The city of Seoul with 10 million population is offering incentives including an annual vehicle tax discount to increase the participation of Weekly No-driving Day (WND) program, a voluntary TDM program encouraging drivers to leave their cars home at least one weekday a week. The compliance of the program rule is monitored by Radio-Frequency IDentification (RFID) systems. In this study, to check the efficiency of the RFID monitoring system, the flow capturing location model is utilized to evaluate the adequateness of the RFID reader locations. Also, this paper proposes an optimal detection rate for the WND program based on economic evaluation results in consideration of costs and benefits of the program.
\end{abstract}

Keywords: travel demand management; monitoring; location problem; flow; Seoul.

Reference to this paper should be made as follows: Ko, J.; Kim, D. K.; Sin, H. G.; Lee, S. 2014. The efficiency of vehicle monitoring locations for a voluntary travel demand management program, Transport 29(3): 326-333.

http://dx.doi.org/10.3846/16484142.2014.953206

\section{Introduction}

As climate has change and severe air pollution become life-threatening, policy makers pay more attention to Travel Demand Management (TDM) as a way to reduce dependency on cars. Concerns about TDM arose from the recognition that there are problems and limits in the transportation supply approach such as a new highway construction. Especially, in such a high density city as Seoul, there is little land available for new transportation facility constructions. Furthermore, the necessity of TDM is increasing due to the budget constraints during years-long recession. TDM, which has been applied in cities around the world, includes various strategies such as congestion pricing, parking management and the provision of high capacity transit. The city of Seoul with 10 million people and about 3 million automobiles has also applied various TDM strategies since late 1990's due to severe traffic congestion (Ko, Cho 2009; Ko 2013). In order to apply TDM effectively, the 'carrot and stick' approach is useful (Meyer 1999). Specially, the efficiency of TDM programs can be maximized when citizens' voluntary participation in the programs is active, which can be motivated by incentives (Brownstone, Golob 1992; Taylor 2007; Hensher, Puckett 2007).

Examples of voluntary TDM policies are carpool started from 1970's (Li et al. 2007) and voluntary travel behavior change schemes, a household-based behavior change technique primarily administered in Europe and Australia (Brög et al. 2009; Taylor 2007; Taylor, Ampt 2003). The effectiveness of the programs was closely monitored and they were generally accepted as an effective way to reduce car dependency (Zhang et al. 2013; Stopher et al. 2009). In the case of Seoul, a voluntary TDM program called the Weekly No-driving Day (WND) program began in 2003 to mitigate traffic congestion in particular in the downtown area (Ko, Cho 2009). In order to motivate more people to participate in the WND program, Seoul Metropolitan Government (SMG) offers various incentives such as discounts of annual vehicle tax, annual traffic impact fee, congestion pricing, and public parking fee. However, such incentives should not be offered to drivers who do not keep the rule of the WND program because public money should not be wasted for no gain. Therefore, in 2006,

Corresponding author: Seungjae Lee

E-mail: sjlee@uos.ac.kr 
SMG introduced a WND monitoring system that requires the WND program participants to attach a RadioFrequency IDentification (RFID) tag on the windshield of their vehicles, and checks the WND program compliance rate by RFID readers installed on major highways. However, since it is not possible to install RFID readers on all roads due to a budget constraint, the monitoring system has a limitation to track all the participating vehicles, implying the importance of determining optimal locations for RFID readers.

Under this circumstance, this paper evaluates the efficiency of current and future RFID reader locations utilizing the Flow Capturing Location Model (FCLM) proposed by Hodgson (1990). More specifically, this paper seeks optimal RFID reader locations that maximize the rate of detection for the WND program participating vehicles with a minimal cost in consideration of the spatial distribution and travel patterns of the WND program participating vehicles. It is expected that the evaluation framework presented in this paper may benefit a future voluntary TDM program monitoring system development.

\section{Weekly No-Driving Day Program}

The WND program, one of voluntary TDM programs, has been implemented in Seoul, South Korea since 2003. Typical passenger car demand control programs force people not to drive according to the last digit of vehicle license plate numbers. Unlike the typical programs, the participants of the WND program can choose one day of a week as their no-driving day considering their car use patterns. To encourage more people (especially, choice riders rather than captive riders) to participate in this program, SMG has offered incentives such as a $5 \%$ reduction in annual vehicle tax, a $50 \%$ discount on congestion charge, and about $10 \%$ to $20 \%$ discount on public parking fees. At the same time, a system composed of RFID tags attached on the windshield of the program participants' vehicles and tag readers installed on major arterials has been utilized to monitor the WND program. Currently, 19 vehicle monitoring stations are operated by SMG for verifying if the participating vehicles comply the no-driving day rule as can be seen in Fig. 1. Based on the verification, the incentives are not provided to the participants who violate the rule more than two times a year.

As of September 30, 2013, the total number of the program participants is 781066 that accounts for about $35 \%$ of total number of eligible passenger cars (noncommercial cars that can carry less than 11 passengers) registered in Seoul (SMG 2013). A previous study conducted by Ko and Cho (2009) investigated the effects of the WND program by two surveys that were to measure the WND program compliance rate and to identify the program participants' characteristics. The WND program compliance rate survey was done for five weekdays and revealed that the day-by-day RFID tag attachment rate and compliance rate for sampled 5884 vehicles were

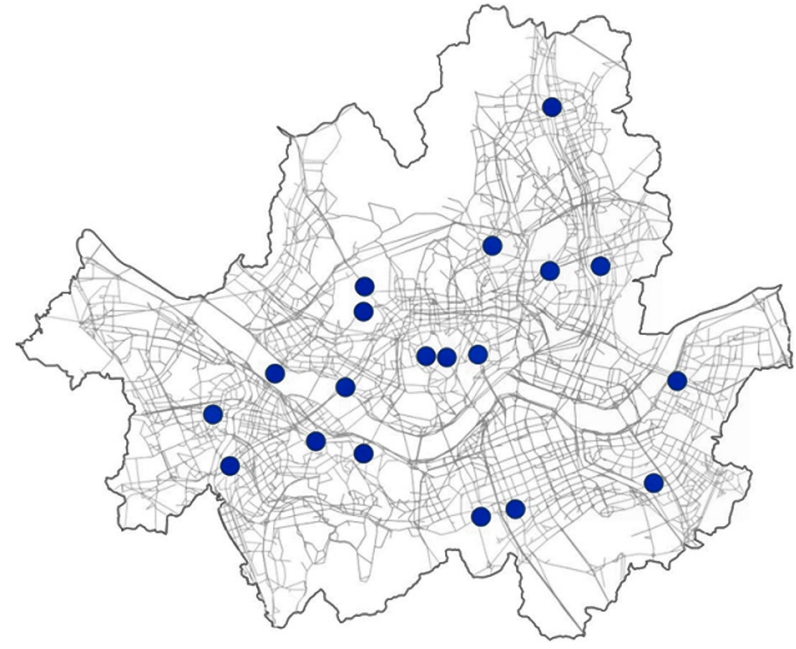

Fig. 1. Current RFID reader locations

$37.5 \%(2209 / 5884)$ and $21.1 \%(1244 / 5884)$, respectively. A recent study found that the estimated rates of tag attachment and compliance were $45.7 \%$ (1295/2832) and $26.1 \%$ (739/2832), respectively, showing potentially better effectiveness compared to the previous study (SMG 2013). These findings suggest that a monitoring system is a crucial element for the success of the voluntary program with the incentives being properly provided to the participants complying with the program rule. Their study also found that the program participation is affected by individuals' characteristics such as the degree of car dependency and age.

\section{Location Problems and a Flow Capturing Model}

\subsection{Location Problems}

The efficiency of the current RFID reader locations can be evaluated by checking whether their locations are adequate considering the travel patterns of the participants' vehicles. In this sense, the evaluation process is likely to involve seeking solutions for optimally locating RFID readers. General location problems can be roughly divided into four categories: set covering problem, maximum covering problem, $\mathrm{p}$-center problem and $\mathrm{p}$-median problem (Daskin 1995). By solving the problems, the systems of facilities and the demand allocated to each facility can be determined. The methodology for general location problems is a good starting point for selecting RFID reader installment sites among numerous candidate locations. However, the general location problem methodology appears inappropriate since it assumes that the facilities are to serve the demand at nodes. This assumption requests special trips between the demand nodes and facilities, which may not be applicable for detecting vehicles traveling on roads. A RFID reader should serve the demand composed of vehicle travel paths through a network. Thus, the demand should be expressed as traffic flows as in the case of billboards.

A simple approach for locating such facilities is to select sites with a sufficient amount of traffic flows, but the approach fails to deal with a potentially damaging 
self-competition problem. Traffic flow on a network may be served by several facilities located close together or on common paths. This multiple covering may result in more occurrences of unserved traffic. As a variant of maximum covering problem, Hodgson (1990) proposed the FCLM, in which $p$ facilities are located so as to intercept as many trips as possible. After the seminal work, the FCLM was further developed and applied to various location problems (Averbakh, Berman 1996; Berman 1997; Berman, Krass 1998; Berman et al. 1992, 1995; Hodgson, Rosing 1992; Hodgson et al. 1996a, 1996b; $\mathrm{Wu}$, Lin 2003). Recently, the FCLM was extended to locate charging facilities for alternative fuel vehicles with a limited driving range, resulting in a flow-refueling location model (Kuby, Lim 2005, 2007; Kuby et al. 2009). These location models efficiently eliminate the self-competition issue by adopting flow-based demand.

\subsection{Flow Capturing Location Model}

The FCLM model maximizes the number of trips that can be potentially detected by a given number of RFID readers $p$ (Hodgson 1990). Thus, the objective function is:

$$
\max Z=\sum_{q \in Q} f_{q} \cdot y_{q}
$$

subject to:

$$
\begin{aligned}
& \sum_{k \in L_{q}} x_{k} \geq y_{q} \text { for all } q \in Q ; \\
& \sum_{k \in K} x_{k}=p,
\end{aligned}
$$

where: $q$ - a particular Origin and Destination (OD) pair; $Q$ - the set of all OD pairs; $f_{q}$ - the flow of WND participants' vehicles between OD pair $q ; k$ - a potential link which RFID tag reader is located; $K$ - the set of all potential links which RFID tag readers are located; $L_{q}-$ the set of links capable of capturing $f_{q} ; p$ - the number of RFID tag readers to be located;

$$
\begin{aligned}
& y_{q}= \begin{cases}1 & \text { if } f_{q} \text { is captured; } \\
0 & \text { if not; }\end{cases} \\
& x_{k}= \begin{cases}1 & \text { if there is a RFID tag reader at link } k ; \\
0 & \text { if not. }\end{cases}
\end{aligned}
$$

This formulation is structurally identical to the maximum covering problem. The first constraint indicates that the flow on path $q$ can be captured only if there is at least one RFID reader on path $q$. The second constraint means that exactly $p$ RFID readers are located. For simplicity, all flows for an OD pair are assumed to take the same path. Hodgson (1990) showed that the FCLM efficiently locates facilities to maximize traffic flow capture by considering all facilities as a system rather than on a facility-by-facility basis.

The model can be solved by stepwise location decisions. At the first round, the solution simply locates a RFID tag reader at the most heavily traveled link, and then identifies non-captured flows by removing the cap- tured flows from consideration. Then, the solution again selects the link most heavily traveled by non-captured flow in the previous round. This procedure is repeated until $p$ facilities are located. This study locates RFID readers on links rather than at nodes. Although a facility installed at a node can theoretically capture all flows passing through the node, the limited communication range between a RFID tag and a reader does not always allow this.

\section{Analysis}

\subsection{Data}

In order to determine RFID reader locations, it is necessary to know the current WND program participants' spatial distribution and travel patterns. As of September, 2013 , the total number of WND program participants is 781066 according to the WND program database. Fig. 2 shows the distribution of the WND program participants by the smallest administrative unit called 'Dong. Overall, it is found that citizens near the city boundary are more likely to participate in the WND program than citizens in downtown areas.

To find the optimal locations for RFID readers, it is necessary to identify the travel paths of the WND program participating vehicles. In fact, however, it is impossible to know every single vehicle's travel path in detail. Therefore, this study assumed that their travel pattern is same as the pattern in the Seoul Travel Demand Model (STDM). In other words, the OD flow patterns of the WND program participating vehicles are assumed to follow the ones in the STDM. Based on the assumption, the authors estimated trips between two zones using the following formula:

$$
\operatorname{Trip}_{i j}=\mathrm{Car}_{i} \cdot \frac{S I_{-}{ }_{\text {Trip }}}{\sum_{j} S I_{-} \operatorname{Trip}_{i j}} \cdot R,
$$

where: $\operatorname{Trip}_{i j}$ - WND program participating vehicle trips between zones $i$ and $j$; $\mathrm{Car}_{i}$ - number of

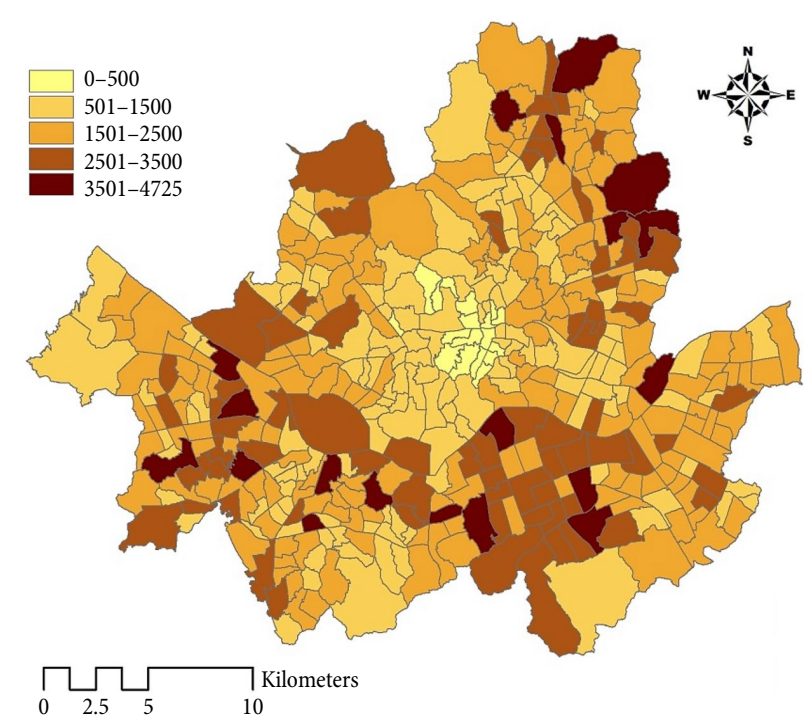

Fig. 2. Distribution of the WND program participants 
the WND program participating vehicles in zone $i$; $S I_{-}$Trip $_{i j}$ - vehicle trips between zones $i$ and $j$ in the STDM; $\sum_{j} S I_{-} T r i p_{i j}$ - number of vehicle trips produced in zone $i$ in the STDM; $R$ - average number of trips made by a passenger car

In the equation, 2.47 was applied for the value of $R$ (SMG 2013). Based on the above OD distribution results, the estimated trips were assigned on the shortest paths of the STDM network with 424 centroids and 19959 links utilizing Dijkstra's algorithm, a graph search algorithm. Note that the simple algorithm generates only a single path for an OD pair, which may not fully reflect real world conditions. However, more realistic algorithms, thus more complex procedures require computational burden as they are likely to generate a tremendously large number of paths. In fact, for the network with 424 centroids, even the simple procedure generates 179776 paths. Future studies are encouraged to apply more realistic approaches for the procedure of

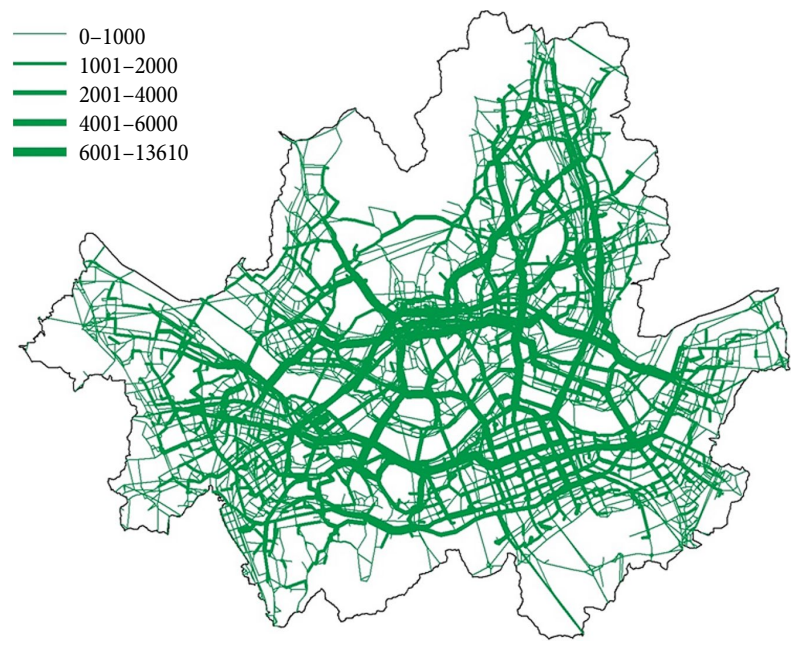

Fig. 3. Estimated link flows of the WND participants' vehicles in Seoul trip assignment. In the assignment process, intra-zonal trips were excluded, with a total number of 524683 interzonal trips being assigned. Fig. 3 shows the estimated link flows of the WND participants' vehicles on the STDM network.

\subsection{Locations of RFID Readers and Their Effectiveness}

Through the flow capturing processes, top two locations (links) where the RFID system can most efficiently monitor the program participating vehicles were identified as shown in Fig. 4. The first location is in the downtown of Seoul with an estimated detection rate of $2.6 \%$. In other words, $2.6 \%$ of the WND program participating vehicles can be detected in the location. The detection rate can be increased up to $4.8 \%$ with the addition of the second location.

Detection rates were estimated by varying the number of installed RFID readers as shown in Fig. 5 . The detection rate becomes $10 \%$ when the RFID systems are installed on 5 locations and it increases up to $50 \%$ with 62 locations. The figure suggests that 395 RFID reader locations are required to obtain a $90 \%$ detection rate and 1252 locations for a $100 \%$ detections rate. As expected, since the marginal detection rate gradually decreases with more RFID reader locations, excessive reader locations may not be economically viable.

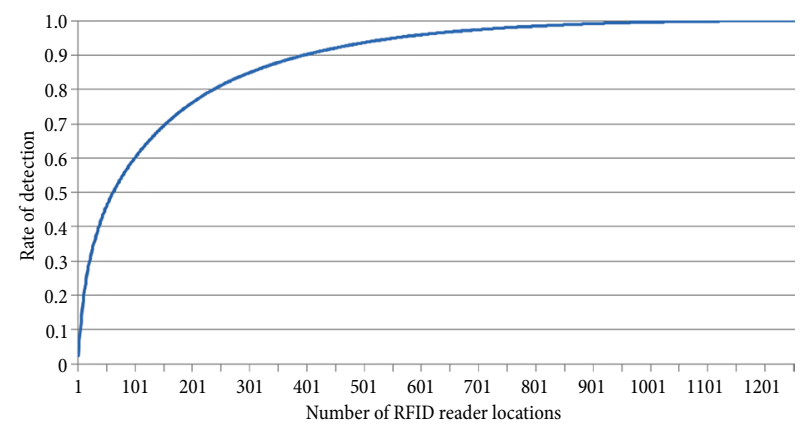

Fig. 5. Number of RFID readers and detection rates a) Top 1 location

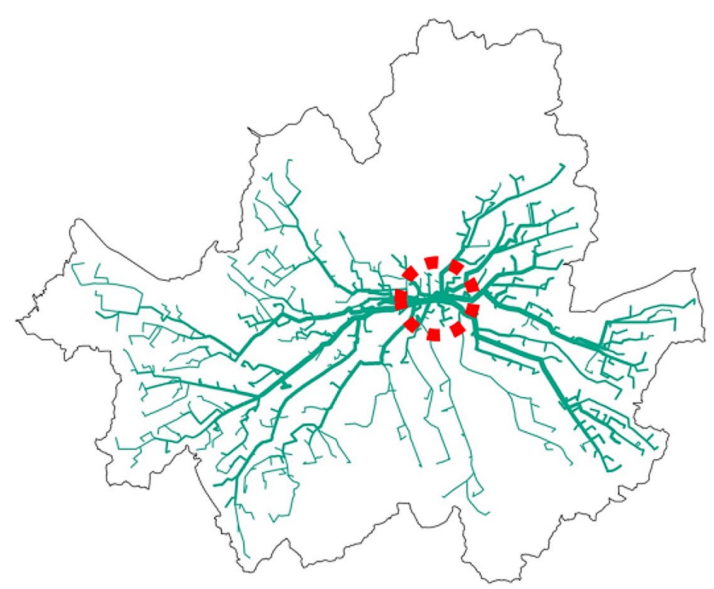

b) Top 2 location

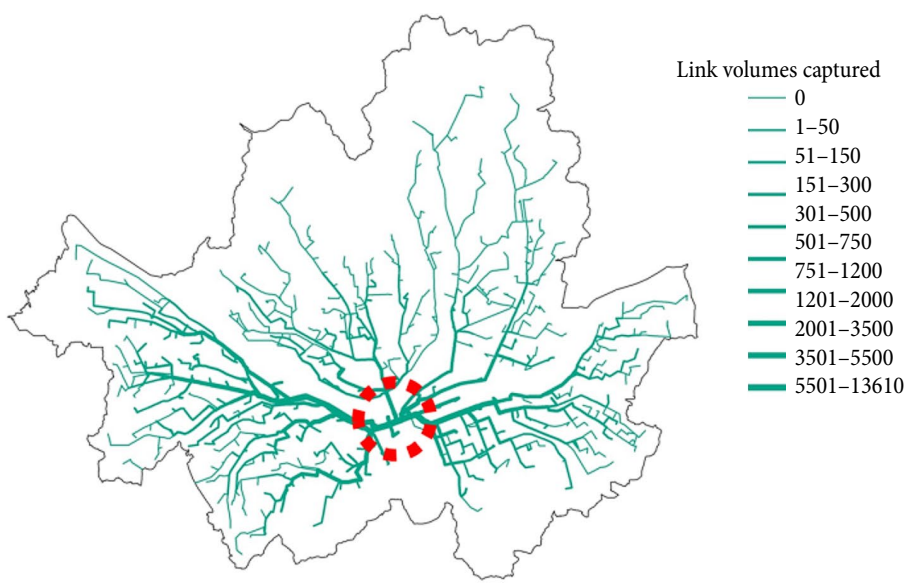

Fig. 4. Top 2 locations that capture the most flows 


\subsection{Evaluating the Efficiency of the Current RFID Reader Locations}

The efficiency of the current 19 RFID reader locations was evaluated, resulting in an estimated detection rate of $7.8 \%$. Note that as suggested in the previous section, only five optimal reader locations can allow a detection rate of $10 \%$. Thus, it appears that the current monitoring system with RFID readers installed on 19 locations is inefficient. In addition to the existing 19 locations, 16 more reader locations (total 35 detector locations) are required to achieve a detection rate of $30 \%$ while only 21 locations are required for the same detection rate if the existing 19 locations are not considered. To obtain a detection rate of $90 \%, 403$ locations are required when considering the existing 19 locations, but it is 395 locations without considering the existing locations.

Table 1 and Fig. 6 show the number of reader locations to obtain target detection rates with and without considering the existing reader locations. As discussed earlier, since the current RFID readers were not installed on optimal locations, additional detectors are required to obtain the same level of detection rate at the optimal locations. As the level of detection rate goes up, however, the difference of detection rates between with and without considering the existing detector locations becomes smaller. It appears that the utility of the existing reader locations also goes up as more detectors are required to obtain higher detection rates. Thus, the effort to find optimal reader locations is essential especially when there is a certain limit in the number of readers due to the budget constraints.

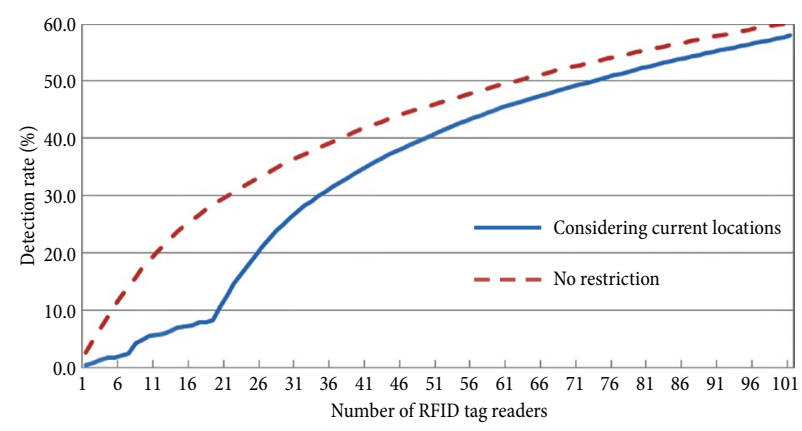

Fig. 6. Number of RFID readers and detection rates

\subsection{Assessment of Cost Effectiveness}

It is natural that the detection rates will increase with more RFID readers. Considering budget constraints, however, it is necessary to evaluate whether more RFID readers are needed in terms of cost effectiveness. In order to evaluate the more RFID option, it was assumed that the more RFID option results in a higher compliance rate which means more traffic volume reductions, and thus more social benefits. Based on this assumption, a cost-benefit analysis was implemented.

The social benefit of reducing one vehicle trip in Seoul was estimated to be $2621846 \mathrm{KRW}$ (about 2500 USD) annually when traffic volume is reduced by $5 \%$ compared to the current level (SMG 2013). The social benefit includes the cost reductions of travel time, vehicle operations, accidents, and air pollutions. Thus, the social benefit was simply computed by multiplying the number of reduced vehicle trips and the monetized social benefit per vehicle trip as below:

\section{Social benefit $=$ Reduced vehicle trips $\times$ \\ Amount of social benefit per vehicle trip.}

In this approach, the amount of social benefit per vehicle trip is set constant (i.e., 2.6 million KRW), by simply assuming that the social benefit is not affected by traffic congestion level. Although congestion cost by one car trip is to be varied depending on the referenced traffic conditions due to by the non-linear relationship between traffic volume and speed, this simplification renders the computation significantly easy. In order to examine how the approach with the constant social benefit by one car trip reduction affects the result of benefit estimation, the range of the car trip reduction effect by the WND program was computed. As a result, the current level of the car trip reduction effect was found to be about $5 \%(0.2 \times 0.46 \times 0.57)$ and it can increase up to $8 \%$ $(0.2 \times 0.46 \times 0.90)$ when the compliance rate becomes $90 \%$, showing a rather narrow variation. This examination suggests that the assumption of the constant social benefit per vehicle trip may not critically influence the benefit estimations. In addition, it is found that the benefit by one vehicle trip reduction decreases to 2.58 million $\mathrm{KRW}$ when the trip reduction rate becomes $10 \%$. This is

Table 1. Required number of RFID reader locations for achieving the target detection rate

\begin{tabular}{|c|c|c|c|c|c|}
\hline \multirow{2}{*}{ Target detection rate } & \multicolumn{2}{|c|}{ Number of locations required } & \multirow{2}{*}{ Difference $(A-B)$} & \multicolumn{2}{|c|}{ Ratio } \\
\hline & Considering current locations $(A)$ & No restriction $(B)$ & & $A /(A-B)$ & $B /(A-B)$ \\
\hline $10 \%$ & 20 & 5 & 15 & 0.75 & 3.00 \\
\hline $20 \%$ & 26 & 11 & 15 & 0.58 & 1.36 \\
\hline $30 \%$ & 35 & 21 & 14 & 0.40 & 0.67 \\
\hline $40 \%$ & 49 & 37 & 12 & 0.24 & 0.32 \\
\hline $50 \%$ & 73 & 62 & 11 & 0.15 & 0.18 \\
\hline $60 \%$ & 110 & 100 & 10 & 0.09 & 0.10 \\
\hline $70 \%$ & 164 & 154 & 10 & 0.06 & 0.06 \\
\hline $80 \%$ & 247 & 237 & 10 & 0.04 & 0.04 \\
\hline $90 \%$ & 403 & 395 & 8 & 0.02 & 0.02 \\
\hline $100 \%$ & 1268 & 1252 & 16 & 0.01 & 0.01 \\
\hline
\end{tabular}


only $1.5 \%$ difference ( 2.58 million vs. 2.62 million KRW) compared to the $5 \%$ reduction case. Consequently, at the current level of traffic conditions in Seoul and a narrow range of traffic volume reductions (from 5 to $8 \%$ ), the assumption of the constant social benefit per vehicle trip reduction seems not to be critical for the benefit cost analysis. Note that, however, the constant amount of benefit may not be justified when the amount is sensitive to traffic conditions and the range of traffic volume changes is wider. It is advised that the characteristics of the network of interest should be carefully examined before applying the simple approach used in this study.

The reduced vehicle trips by the WND program, $E$ can be calculated by the following formula:

$$
E=0.2 \cdot W \cdot A \cdot D \cdot R,
$$

where: $W$ - number of WND program participants; $A$ RFID tag attachment rate; $D$ - WND program compliance rate.

In above formula, ' 0.2 ' is a coefficient that reflects the program characteristics: the WND program participants should not drive on a day out of five working days every week. As of September, 2013, the values for $W$ and $A$ in above formula are 781066 and 0.46. (SMG 2013). The value of $R$ is 2.47 as in the previous equation. The WND program compliance rate, $D$ was considered as a function of the number of the RFID reader locations because the program participants are more likely to observe the rule when they are more monitored. It is assumed that there is a simple linear relationship between the WND program compliance rate and the number of the RFID reader locations. Based on this assumption, the compliance rate, $D$, was estimated by the following formula:

$$
D=D_{0}+\left(D_{1}-D_{0}\right) \cdot \frac{x-x_{0}}{x_{1}-x_{0}} .
$$

In the above formula, $D_{0}$ and $x_{0}$ are the current levels of compliance rate and detection rate, respectively. A recent survey (SMG 2013) identified that the current compliance rate is 0.57 , and thus this study utilized the values of 0.57 and 0.078 for $D_{0}$ and $x_{0}$, respectively. Concerning the values for $D_{1}$ and $x_{1}$, they are assumed to be 0.9 and 1.0 respectively under the supposition that the maximum WND program compliance rate is limited to $90 \%$ even with a detection rate of $100 \%$.

Items considered in the calculation of WND program operation costs are as follows (SMG 2013):

- annual operation costs and incentives (such as annual vehicle tax discount) for the WND program (11.2 billion KRW; about 10.7 million USD);

- RFID reader installation cost (100 million KRW (about 95000 USD) per location) and maintenance cost (10 million KRW (about 9500 USD) per year, $10 \%$ of the installation cost).

The benefit to cost $(B / C)$ ratio was calculated with a discount rate of $4.5 \%$ under the assumption that the life of the RFID reader lasts 10 years. As a result, $B / C$

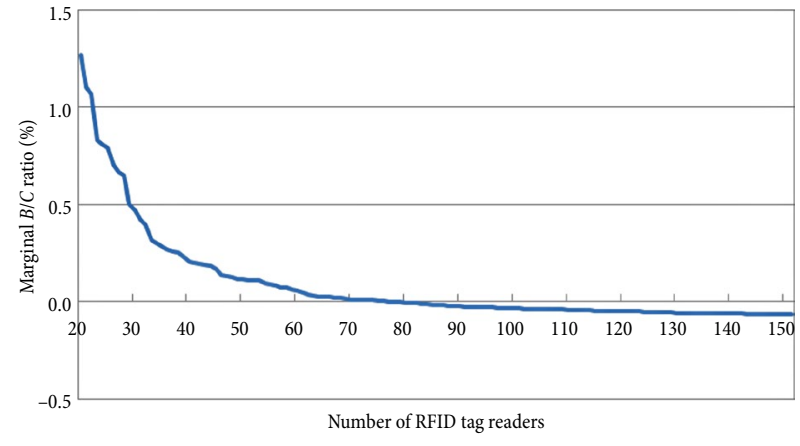

Fig. 7. Number of RFID reader locations and marginal ratio of benefit to cost

ratio was found to be over 1.0 even when RFID readers are installed on 1252 locations of which detection rate is $100 \%$. However, as shown in Fig. 7, marginal $B / C$ ratio increases up to a certain point and then decreases after the point as the number of RFID reader locations increases. In detail, marginal $B / C$ ratio starts to decrease after the installation of RFID readers on 77 road links of which estimated detection rate is $51.4 \%$. In other words, the WND program benefit compared to the program cost will be maximized by installing RFID readers on 77 road links. Referentially, the maximized point of marginal $B / C$ ratio was found to increase up to 85 road links when $95 \%$ is applied for $D_{1}$, the assumed maximum compliance rate of the WND program.

\section{Conclusions}

As many people are concerned about sustainable urban transportation systems, TDM is getting more attention as a way to reduce automobile dependency. Especially, voluntary participation by offering incentives as a way of implementing TDM has been applied in many cities in recent years. In 2003, the city of Seoul with a 10 million population initiated the WND program which requires participants not to use their vehicles for one working day a week. The SMG is offering incentives such as discounts of annual vehicle tax, annual traffic impact fee, congestion charge, and public parking fee to encourage WND program participation. These incentives are offered as long as the program participants do not violate the rule more than two times a year. For monitoring the compliance of the rule, the RFID-based system composed of RFID tags attached on the windshield of the participants' vehicles and RFID readers installed on major highways is now under operation.

In this study, as a task to examine the efficiency of the RFID monitoring system, the FCLM was utilized to estimate the detection rate achieved by the existing RFID reader locations. Analysis results showed that the estimated detection rate is only $7.8 \%$ for the existing 19 RFID reader locations. However, if five optimal detector locations were selected regardless of existing 19 locations, then the detection rate can be $10 \%$. In consideration of this finding, the optimal RFID reader location selection appeared to be very important especially when 
we should minimize the number of RFID reader locations. Moreover, the results revealed that the more RFID readers are installed, the less marginal detection rates were obtained.

For more effective WND program monitoring, it is necessary to increase the number of RFID reader locations even if the readers are fake ones that warn participating drivers. As discussed earlier, we need to find an optimal number of RFID reader locations in consideration of installation and maintenance costs. The results of an economic evaluation considering the cost together with benefits from the program showed that the maximum operation utility can be achieved at a detection rate of about $50 \%$. Note that this result, however, can be affected by numerous factors such as the levels of compliance rates associated with detection rates and system installation and operation costs. Thus, cautions should be exercised when this methodology is applied in other cities.

It is expected that the evaluation methodology presented in this paper may be beneficial to other cities considering incentive-based TDM programs requiring efficient monitoring systems. Nevertheless, this study has a limitation in that the WND program participants' travel pattern was assumed to be the same as that of general drivers. As suggested by the previous research efforts, the travel pattern or the degree of car dependency of the voluntary TDM program participants may be quite different from that of non-participants (Ko, Cho 2009). In addition, it is little known about how the monitoring system affects the compliance rate of the TDM program. The sound understanding about such behavior would improve the accuracy of the monitoring system evaluations.

\section{Acknowledgments}

This work was supported by the National Research Foundation of Korea (NRK) grant funded by the Korea Government (MEST), NRF-2014R1A2A2A01005155.

\section{References}

Averbakh, I.; Berman, O. 1996. Locating flow-capturing units on a network with multi-counting and diminishing returns to scale, European Journal of Operational Research 91(3): 495-506. http://dx.doi.org/10.1016/0377-2217(94)00369-6

Brownstone, D.; Golob, T. F. 1992. The effectiveness of ridesharing incentives: discrete-choice models of commuting in Southern California, Regional Science and Urban Economics 22(1): 5-24.

http://dx.doi.org/10.1016/0166-0462(92)90023-T

Berman, O. 1997. Deterministic flow-demand location problems, The Journal of the Operational Research Society 48(1): 75-81. http://dx.doi.org/10.2307/3009946

Berman, O.; Krass, D. 1998. Flow intercepting spatial interaction model: a new approach to optimal location of competitive facilities, Location Science 6(1-4): 41-65. http://dx.doi.org/10.1016/S0966-8349(98)00047-3

Berman, O.; Krass, D.; Xu, C. W. 1995. Locating flow-intercepting facilities: new approaches and results, Annals of Operations Research 60(1): 121-143. http://dx.doi.org/10.1007/BF02031943
Berman, O.; Larson, R. C.; Fouska, N. 1992. Optimal location of discretionary service facilities, Transportation Science 26(3): 201-211. http://dx.doi.org/10.1287/trsc.26.3.201

Brög, W.; Erl, E.; Ker, I.; Ryle, J.; Wall, R. 2009. Evaluation of voluntary travel behaviour change: experiences from three continents, Transport Policy 16(6): 281-292.

http://dx.doi.org/10.1016/j.tranpol.2009.10.003

Daskin, M. S. 1995. Network and Discrete Location: Models, Algorithms, and Applications. John Wiley \& Sons, Inc. http://dx.doi.org/10.1002/9781118032343

Hensher, D. A.; Puckett, S. M. 2007. Congestion and variable user charging as an effective travel demand management instrument, Transportation Research Part A: Policy and Practice 41(7): 615-626. http://dx.doi.org/10.1016/j.tra.2006.07.002

Hodgson, M. J. 1990. A flow-capturing location-allocation model, Geographical Analysis 22(3): 270-279. http://dx.doi.org/10.1111/j.1538-4632.1990.tb00210.x

Hodgson, M. J.; Rosing, K. E. 1992. A network location-allocation model trading off flow capturing and $p$-median objectives, Annals of Operations Research 40(1): 247-260. http://dx.doi.org/10.1007/BF02060480

Hodgson, M. J.; Rosing, K. E.; Leontien, A.; Storrier, G. 1996a. Applying the flow-capturing location-allocation model to an authentic network: Edmonton, Canada, European Journal of Operational Research 90(3): 427-443. http://dx.doi.org/10.1016/0377-2217(95)00034-8

Hodgson, M. J.; Rosing, K. E.; Zhang J. 1996b. Locating vehicle inspection stations to protect a transportation network, Geographical Analysis 28(4): 299-314. http://dx.doi.org/10.1111/j.1538-4632.1996.tb00937.x

Ko, J. 2013. Vehicle trip generation rates for office buildings under urban settings, ITE Journal 8(2): 41-45.

Ko, J.; Cho, Y. 2009. Voluntary program to reduce car use: weekly no-driving day in Seoul, South Korea. Transportation Research Record 2118: 1-7. http://dx.doi.org/10.3141/2118-01

Kuby, M.; Lim, S. 2005. The flow-refueling location problem for alternative-fuel vehicles, Socio-Economic Planning Sciences 39(2): 125-145. http://dx.doi.org/10.1016/j.seps.2004.03.001

Kuby, M.; Lim, S. 2007. Location of alternative-fuel stations using the flow-refueling location model and dispersion of candidate sites on arcs, Networks and Spatial Economics 7(2): 129-152. http://dx.doi.org/10.1007/s11067-006-9003-6

Kuby, M.; Lines, L.; Schultz, R.; Xie, Z.; Kim, J.-G.; Lim, S. 2009. Optimization of hydrogen stations in Florida using the flow-refueling location model, International Journal of Hydrogen Energy 34(15): 6045-6064.

http://dx.doi.org/10.1016/j.ijhydene.2009.05.050

Li, J.; Embry, P.; Mattingly, S. P.; Sadabadi, K. F.; Rasmidatta, I.; Burris, M. 2007. Who chooses to carpool and why?: examination of Texas carpoolers, Transportation Research Record 2021: 110-117. http://dx.doi.org/10.3141/2021-13

Meyer, M. D. 1999. Demand management as an element of transportation policy: using carrots and sticks to influence travel behavior, Transportation Research Part A: Policy and Practice 33(7-8): 575-599. http://dx.doi.org/10.1016/S0965-8564(99)00008-7 
SMG. 2013. Evaluation of Weekly No-Driving Day Program. Seoul Metropolitan Government (SMG).

Stopher, P.; Clifford, E.; Swann, N.; Zhang, Y. 2009. Evaluating voluntary travel behaviour change: suggested guidelines and case studies, Transport Policy 16(6): 315-324. http://dx.doi.org/10.1016/j.tranpol.2009.10.007

Taylor, M. A. P. 2007. Voluntary travel behavior change programs in Australia: the carrot rather than the stick in travel demand management, International Journal of Sustainable Transportation 1(3): 173-192. http://dx.doi.org/10.1080/15568310601092005

Taylor, M. A. P.; Ampt, E. S. 2003. Travelling smarter down under: policies for voluntary travel behaviour change in Australia, Transport Policy 10(3): 165-177. http://dx.doi.org/10.1016/S0967-070X(03)00018-0

Wu, T.-H.; Lin, J.-N. 2003. Solving the competitive discretionary service facility location problem, European Journal of Operational Research 144(2): 366-378. http://dx.doi.org/10.1016/S0377-2217(01)00391-5

Zhang, Y.; Stopher, P.; Halling, B. 2013. Evaluation of SouthAustralia's TravelSmart project: changes in community's attitudes to travel, Transport Policy 26: 15-22.

http://dx.doi.org/10.1016/j.tranpol.2012.06.008 